\title{
Fentanyl buccal tablet for breakthrough cancer pain in clinical practice: results of the non-interventional prospective study ErkentNIS
}

\author{
Eva Katharina Masel $^{1} \cdot$ Robert Landthaler $^{2} \cdot$ Margit Gneist $^{3} \cdot$ Herbert Hans Watzke $^{1}$
}

Received: 20 April 2017 / Accepted: 15 August 2017 / Published online: 28 August 2017

(C) The Author(s) 2017. This article is an open access publication

\begin{abstract}
Purpose Several patients with advanced cancer suffer from breakthrough cancer pain (BTcP). BTcP is pain exacerbation despite opioid baseline therapy. Fentanyl buccal tablet (FBT) is a rapid-onset opioid for the treatment of BTcP. The aim of this study is to document the feasibility of FBT in patients with BTcP. Methods The study was performed in 64 centers. Basic pain score was rated on a numeric rating scale (NRS) before and after treatment. BTcP episodes, baseline opioid therapy, and FBT dose were rated as well as individual dose titration, findings on tolerability, patient satisfaction, and safety of the drug. Results Two hundred sixty-three patients were available for analysis. Patients rated a basic pain score of 6 (range 2-10) points on an NRS and described an average of 2 to $5 \mathrm{BTcP}$ episodes per day. After titration of FBT, BTcP control was achieved within $5 \mathrm{~min}$ in $36 \%$, within $10 \mathrm{~min}$ in $68 \%$, and within $15 \mathrm{~min}$ in $95 \%$. Basic pain score decreased to a mean NRS of 4 and BTcP episodes decreased to $<1$ to 3 episodes per day. BTcP control, onset of action of FBT, potency of FBT, tolerability of FBT, and safety of FBT were rated as excellent or good by 89 to $99 \%$ of the patients. Adverse drug reactions were registered in $3 \%$.

Conclusions Treatment with FBT led to rapid pain relief and reductions in the number of $\mathrm{BTCP}$ episodes and patient satisfaction was rated as excellent or good.
\end{abstract}

Eva Katharina Masel

eva.masel@meduniwien.ac.at

1 Division of Palliative Care, Department of Internal Medicine I, Medical University of Vienna, Waehringer Guertel 18-20, 1090 Vienna, Austria

2 Joint Practices for Obstetrics and Gynecology, Krumbach, Germany

3 Teva Ratiopharm Pharmaceutical Industries, Vienna, Austria
Keywords Cancer $\cdot$ Breakthrough cancerpain $\cdot$ Palliative care

\section{Introduction}

Pain is a major complication in patients suffering from advanced cancer. Cancer diseases are frequently associated with physical complaints that severely affect the quality of life of the patients concerned. The prevalence of pain in the case of solid tumors is reported to be between $15 \%$ and more than $75 \%[11,21]$. Pain might be disease related or caused by antineoplastic treatment [2]. However, a high percentage of patients remain insufficiently treated [7, 19]. As distinct from background cancer pain in the form of consistent pain, breakthrough cancer pain $(\mathrm{BTcP})$ is classified as an acute painful episode that occurs in patients already receiving baseline opioids who describe a baseline pain of mild to moderate intensity [5]. Cancer patients experience $\mathrm{BTcP}$ of varying severity and intensity [28]. BTcP has a rapid onset and brief duration, with $64 \%$ of patients reporting the end of a BTcP episode after 30 min $[10,18]$. It negatively affects the quality of life and impairs activities of daily living and mood [22]. According to the high prevalence of pain in oncology and palliative care, individualized pain assessments to differentiate background pain, neuropathic pain, visceral pain, and BTcP should be a standard of care in mitigating suffering and burden of disease. Treatment options consist of opioid-based pharmacotherapy according to the World Health Organization (WHO) analgesic ladder and interventional, radiotherapeutic, and rehabilitative, surgical, or psychological interventions [13]. Since the development of the WHO analgesic ladder, the use of opioids has led to much better pain control, making it possible for many patients to maintain their quality of life $[25,30]$. In the case of BTcP, the analgesic onset of extended-release opioids starts after $30 \mathrm{~min}$, and patients may experience insufficient pain 
relief after their administration. Therefore, BTcP may be more effectively managed by rapid-onset opioids. In clinical practice, rapid-onset opioids still remain unused, and the treatment of BTcP can be described as far from encouraging [29]. Transmucosal fentanyl formulations have been developed to provide analgesia with a rapid onset between 10 and $15 \mathrm{~min}$ $[8,14,23,24,27]$. Fentanyl buccal tablets (FBTs) have been developed to improve cancer pain treatment and have shown to be well tolerated in clinical trials [9]. Patients responding to extended-release opioids, with well-controlled background pain, and having rapid pain onset and frequent cancer BTcP episodes per day, may benefit from rapid acting opioids or rapid-onset opioids such as FBTs. Treatment with rapidonset opioids starts with the lowest dose and is titrated until an effective analgesic effect is reached [6]. The FBT drug Effentora ${ }^{\circledR}$ received marketing approval in April 2008. It is indicated for the treatment of BTcP in adult cancer patients who are already receiving baseline opioid therapy. The active substance fentanyl citrate is rapidly absorbed through the oral mucosa (buccal) or by a sublingual route directly into the blood and thus shows a rapid onset. The aim of this prospective, non-interventional study (NIS) named "ErkentNIS" was to document the tolerability, patient satisfaction, manageability, and safety of the use of FBT in patients suffering from $\mathrm{BTcP}$ in a large patient cohort in real-world clinical practice. ErkentNIS stands for EffentorRa ${ }^{\circledR}$ im KlinischEN AllTag eine Nicht-Interventionelle Studie which is German and signifies "Effentora in clinical routine - a non-interventional study."

\section{Methods}

\section{Study design and patients}

This prospective, open-label, non-interventional trial in a postmarketing section was conducted according to Section 4 (23) paragraph 4 of the Austrian Medicines Act (AMG). The study received institutional review board approval. All participants received full information about the study, were ensured the confidentiality of the collected data, did have written information about the study, did provide written informed consent, and did know that they could refuse their participation or withdraw from the study without consequence.

As a primary objective, adequate and rapid pain relief within $10 \mathrm{~min}$ of application was to be demonstrated for treatment with FBT. FBT was to be uptitrated to an individually effective dose providing for adequate analgesia. Further aims were detailed characterization of BTcP and assessments of tolerability, patient satisfaction, manageability, and safety of FBT. The study was performed by office-based physicians or clinicians, outpatient pain centers, and oncology centers throughout Germany or Austria. The study enrolled adult cancer patients for whom therapy with FBT for the treatment of BTcP was indicated. The patients were to be without any of the contraindications described in the Summary of Product Characteristics (SmPC) and had to already be receiving adequate baseline opioid therapy for the treatment of cancer pain. The patients receiving an adequate baseline opioid therapy included those given at least $60 \mathrm{mg}$ oral morphine daily, at least $25 \mu \mathrm{g}$ transdermal fentanyl per hour, at least $30 \mathrm{mg}$ oxycodone daily, at least $8 \mathrm{mg}$ oral hydromorphone daily, or an analgesic ally equivalent dose of another opioid for a minimum duration of 1 week or longer.

\section{Data collection}

The study was performed in 64 centers in Germany and Austria. Data on patient demographic and clinical characteristics were collected within a 19-month period. The envisaged observation/follow-up period for the individual patient was 8 weeks. Upon inclusion into the study, the treating physician was to perform a screening evaluation (Visit 1). The findings collected were documented in a standardized case report form. After about 4 weeks of follow-up, an interim evaluation (Visit 2) took place. After an overall follow-up time of 8 weeks, a final evaluation was to be done. Overall, three evaluations were scheduled during the time of documentation. Pain relief was measured by a numeric rating scale (NRS) $0-10$.

Visit 1: Baseline documentation (upon initiation of treatment): patient characteristics, basic pain history, opioid baseline therapy, basic pain intensity as reported by patient, assessment of breakthrough pain, etiology, sites, characteristics, causes, and occurrence of breakthrough pain Visit 2: Interim documentation (therapeutic follow-up, after approximately 2-4 weeks): assessment of opioid baseline therapy, rescue medication, average basic pain intensity as reported by patient, breakthrough pain during the last week/prior to start of treatment with FBT, treatment with FBT rating of treatment experiences

Visit 3: Final documentation (after approximately 8 weeks or upon discontinuation of treatment): assessment of opioid baseline therapy, rescue medication to date, average basic pain intensity as reported by the patients, assessment of breakthrough pain, route of administration of FBT, rating of treatment experiences with FBT, titration of FBT versus the respectively last documentation

For assessing baseline opioid therapy, opioid doses were converted to oral morphine equivalents. Equianalgesic conversions were performed with an online calculator (http:// clincalc.com/opioids) based on the American Pain Society guidelines $[1,20]$. 


\section{Statistical analysis}

Statistical methods were limited to descriptive statistics only. We calculated absolute, relative, and adjusted relative (i.e., minus missing data) frequencies for categorical variables and measures of central tendency (mean or median) and spread (minimum and maximum as well as standard deviation or interquartile range) as appropriate for continuous variables for all three time points. We used SAS 9.3 for Windows (SAS Institute, Cary, NC) for all calculations.

\section{Results}

\section{Demographic and clinical characteristics}

Table 1 shows the patients' characteristics. A total of 267 patients at 64 centers was included in the study. A total of 126 (47\%) was male, and 137 (53\%) patients were female. Median age was 66 years (range 26-89). Four patients were excluded from the study, as appropriate consent was missing. The data from 263 patients were available for statistical analysis (Fig. 2). The number of patients per study site varied between 1 and 32 patients. Of the 263 patients originally enrolled in the study, data for $89 \%(n=235)$ of the patients were available for all three evaluation time points. For Visit 2, the data for $94 \%$ $(n=246)$ of patients were available, along with the data for 90\% $(n=236)$ for Visit 3 (Fig. 4). The time of observation or follow-up was 32 days (median: 29, range 6-91).

\section{Etiology and severity of background cancer pain}

The etiology of pain was described as mixed pain by $77 \%$ $(n=203)$ of the patients at the screening evaluation, while $14 \%(n=37)$ of the patients suffered from nociceptive pain, and $9 \%(n=23)$ described neuropathic pain. Patients were to identify their pain before the initiation of baseline opioid therapy on an NRS from 0 to 10 . Median pain intensity score was 6 points (range $2-10$ ).

\section{Treatment of background cancer pain}

Opioid treatment was as follows: $54 \%(n=141)$ of the patients received transdermal fentanyl (oral morphine equivalent 197), $17 \%(n=46)$ of the patients received hydromorphone (oral morphine equivalent 178$), 16 \%(n=41)$ of the patients received oxycodone (oral morphine equivalent 131), 10\% $(n=27)$ of the patients received sustained release morphine (oral morphine equivalent 91), and 3\% $(n=8)$ of the patients received buprenorphine (oral morphine equivalent 100).
Table 1 Patient characteristics

\begin{tabular}{llr}
\hline & Number & $\%$ \\
\hline & 263 & $100 \%$ \\
Sex & 137 & $52 \%$ \\
$\quad$ Female & 126 & $48 \%$ \\
$\quad$ Male & & \\
Age & $66(26-89$ years $)$ & \\
$\quad$ Years, median (range) & $24\left(13-38 \mathrm{~kg} / \mathrm{m}^{2}\right)$ & \\
BMI & & $18 \%$ \\
Tumor disease & 48 & $13 \%$ \\
Breast cancer & 35 & $11 \%$ \\
Prostate cancer & 29 & $4 \%$ \\
Lung cancer & 18 & $47 \%$ \\
Colorectal cancer & 11 & $14 \%$ \\
Pancreatic cancer & 122 & $7 \%$ \\
$\quad$ Other & & \\
Metastatic spread $(n=55)$ & 37 & \\
Bone metastases & 18 & \\
Liver metastases & & \\
\hline
\end{tabular}

\section{Etiology and severity of BTcP}

The etiology of BTcP was described as mixed pain by $74 \%$ ( $n=192)$ of the patients. BTcP had nociceptive characteristics in $15 \%(n=38)$ of the patients and neuropathic characteristics in $11 \%(n=28)$. Before treatment, the duration of BTcP per episode was reported to last between 31 and $60 \mathrm{~min}$ in the majority of the patients (Fig. 1). The maximum pain intensity peak in BTcP episodes was reached after less than $5 \mathrm{~min}$ in $15 \%(n=40)$, after 5 to $10 \mathrm{~min}$ in $31 \%(n=82)$ of the patients, after 11 to $15 \mathrm{~min}$ in $25 \%(n=66)$, after 16 to $30 \mathrm{~min}$ in $13 \%(n=35)$, after 31 to $60 \mathrm{~min}$ in $13 \%(n=35)$, and after more than $60 \mathrm{~min}$ in $3 \%$ $(n=5)$. Pain episodes persisted for less than $5 \mathrm{~min}$ in $3 \%$ $(n=8)$, for 5 to $10 \mathrm{~min}$ in $18 \%(n=47)$, for 11 to $15 \mathrm{~min}$ in $15 \%(n=39)$, for 16 to $30 \mathrm{~min}$ in $22 \%(n=57)$, for 31 to $60 \mathrm{~min}$ in $24 \%(n=62)$ of the patients, and for more than $60 \mathrm{~min}$ in $18 \%$ $(n=39)$. And, for two patients, no data were provided.

\section{Dose titration of FBT and treatment of BTcP}

In $70 \%$ of the patients, $\mathrm{BTcP}$ had already been treated prior to inclusion in the study (Fig. 2). Inadequate efficacy of the previous medication was the most frequent reason for a switch to FBT in $84 \%$ ( $n=156)$ of the patients, followed by inadequate onset of action of the previous medication in $52 \%(n=97)$ or inadequate tolerability of the previous rescue medication in $5 \%(n=10)$ of the patients.

Treatment with FBT was started at an initial dose of $100 \mu \mathrm{g}$ by $69.2 \%(n=182)$ of the patients, and $24.7 \%(n=65)$ of the patients started with an initial dose of $200 \mu \mathrm{g} .4 .2 \%(n=11)$ of the patients used an initial dose of $400 \mu \mathrm{g}$ and $1.9 \%(n=5)$ of the patients a dose of $600 \mu \mathrm{g}$. Although titration of FBT according to SmPC was not required in the observational protocol, $29.5 \%$ ( $n=74)$ of 251 patients, having had no pretreatment with fentanyl, received an initial dose of $200 \mu \mathrm{g}$ or more. In $36.9 \%(n=97)$ 
Fig. 1 Duration of breakthrough pain episodes before FBT versus duration at last observation during FBT therapy $(n=242)$

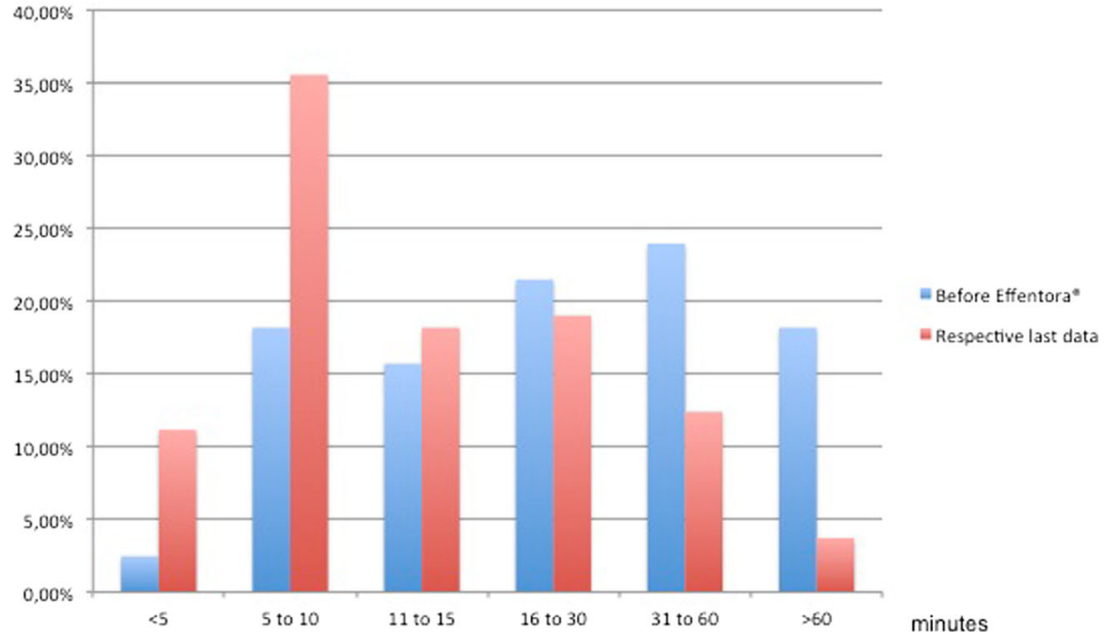

of the patients, the starting dose was at the same time the final dose, while for the remaining 166 patients, up to five dose adjustments were done. For patients having used fentanyl before, up to two dose adjustments were observed. Dose adjustment for FBT usually took 6.2 days ( \pm 11.80 ; median 2.00 ) with a minimum of 0 days (starting dose and dose at the end of titration identical) and a maximum of 92 days.

Patients were to identify their BTcP intensity before initiation of FBT treatment on an NRS from 0 to 10 . The median pain intensity score was 6 points (range 2-10). After successful titration of FBT, adequate BTcP control was achieved within $5 \mathrm{~min}$ in $36 \%$ of the patients, within $10 \mathrm{~min}$ in $68 \%$, and within $15 \mathrm{~min}$ in 95\% (Fig. 3). BTcP pain score decreased to a mean NRS of 4, and $\mathrm{BTcP}$ episodes decreased to $<1$ to 3 episodes per day. BTcP control was rated as excellent or good by $92 \%(n=242)$ of the patients, the onset of action was assessed as excellent or good by $89 \%$ ( $n=234)$ of the patients, and the potency of FBT was rated as excellent or good by $91 \%(n=238)$ of the patients. The tolerability of FBT was assessed as excellent or good by $97 \%$
( $n=253)$ of the patients, and the safety of FBT was considered as excellent or good by $99 \%$ of the patients. Adverse drug reactions were registered in $3 \%$ of all the patients. Six events in $1 \%(n=3)$ of the patients were assessed as serious by the treating physician. These events included the following: (1) general deterioration of physical health status, (2) facial swelling, (3) superinfection, (4) jaundice, (5) epigastric pain, and (6) confusion. With the exception of pain, all of these events were assessed as serious because they were associated with inpatient hospitalization.

\section{Discontinuation of treatment}

Sixteen percent $(n=43)$ of all the patients discontinued treatment. Five percent $(n=12)$ did not finish titration, $3 \%(n=7)$ of the patients discontinued treatment at Visit 2, and $10 \%$ $(n=24)$ of the patients discontinued treatment at Visit 3 (Fig. 2). Most of the reasons for the discontinuation of treatment were "tumor progression" and "death of the patient" in $7 \%(n=19)$ of all the patients followed by "treatment of
Fig. 2 Previous treatment of breakthrough pain $(n=263)$

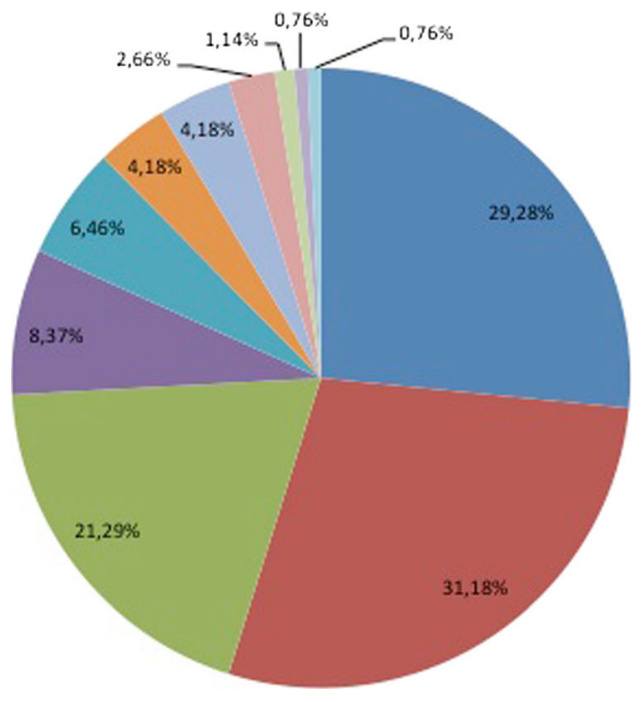

$=$ No treatment

- Non-opioid analgesics

- Immediate release morphine

- Increase of maintenance analgesic

- Immediate release hydromorphone

"Immediate release oxycodone

- Low-potency opioids

= Oral-transmucosal fentanyl

- Finished nasal fentanyl

" Sublingual fentanyl

- Immediate release buprenorphine 

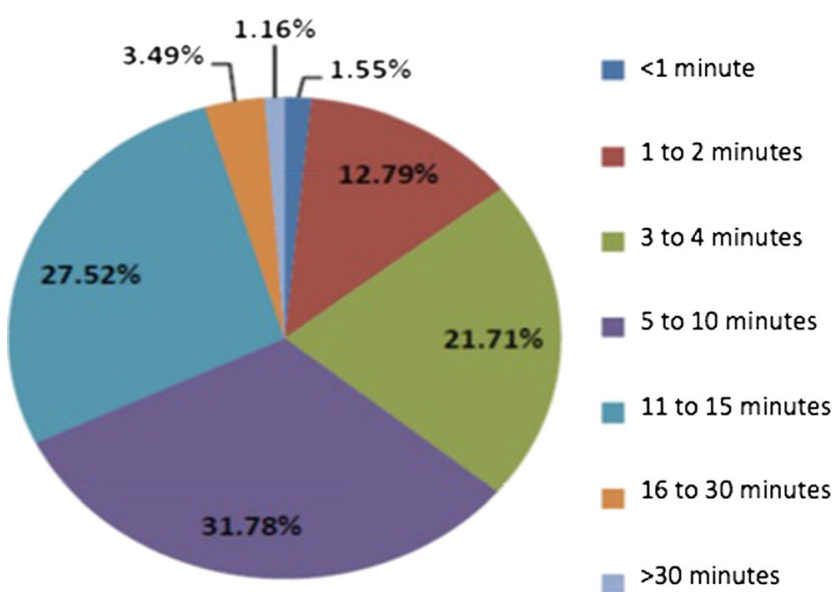

Fig. 3 Duration to onset of analgesic pain relief after successful titration of FBT $(n=258)$

breakthrough pain no longer required" in $3 \%(n=9)$, "upon request of the patient" in $2 \%(n=6)$, and adverse events in $1 \%$ $(n=3)$ of all the patients (Fig. 4).

\section{Discussion}

This NIS was designed to document the tolerability, patient satisfaction, manageability, and safety of the use of the rapidonset opioid FBT in cancer patients suffering from BTcP in real-life clinical practice in a cohort of 263 patients. Pain is a very important cancer-related symptom of high prevalence [21]. Pain periodicity and intensity have to be assessed to differentiate BTcP from background pain or end-of-dose failure, meaning a pain flare at the end of a dosing interval [12]. The results of this study show that FBT as a buccal or sublingual administered rapid-onset opioid has proven to be feasible in clinical practice [4]. In the current study, $71 \%$ of the patients reported maximum pain intensity within 5 to $10 \mathrm{~min}$, which emphasizes the demand for rapid analgesia, and which however illustrates why extended-released opioids might not provide appropriate analgesia. In $71 \%$ of the patients, BTcP had already been treated before the patients' inclusion in the study using up to three different treatments, frequently by means of non-opioid analgesics, short-acting opioids, or by an increase in the dose of the baseline opioid therapy. Inadequate response to the previous rescue medication was the most common reason for a switch to FBT, followed by inadequate onset of action of the previous medication. In the current study, after successful titration of FBT, adequate analgesic BTcP control was achieved within $5 \mathrm{~min}$ in $36 \%$ of the patients, within $10 \mathrm{~min}$ in $68 \%$, and within $15 \mathrm{~min}$ in $95 \%$. BTcP control, onset of action, potency, and tolerability were rated as excellent or good by a majority of the patients. Further clinical trials are needed to better define BTcP and its treatment options [15]. Rapid-acting opioids are approved for opioid-tolerant



Fig. 4 Flow diagram of the study population

adults suffering from BTcP and provide analgesia through rapid onset of pain relief [26]. Previous studies have shown that different formulations of transmucosal immediate-release fentanyl can be used to manage BTcP: buccal soluble film, buccal tablet, intranasal spray, sublingual spray, and sublingual tablet [3]. For the management of BTcP, FBT was shown to be superior to oral morphine [16]. A superior analgesic effect versus oral morphine was also shown for fentanyl pectin nasal spray [17]. Another study demonstrated that patients preferred FBT over immediate-release oxycodone [31]. Treatment strategies have to consider the underlying disease, co-morbidities, pain characteristics, and patient's preferences. Background pain should be controlled by the use of longacting opioid formulations to achieve plasma concentrations that facilitate around-the-clock analgesia [21]. Besides pharmacological treatment, disease-modifying approaches, such as antineoplastic treatment, surgery or radiation therapy, and physical and occupational therapy, may provide pain relief. The limitations of this study have to be addressed. This was an observational study, which solely focused on the feasibility of FBT in clinical practice and did not intend to compare different rapid-onset opioids. When evaluating the efficacy of the study drug before and after administration, changes in BTcP intensity might also have been influenced by other factors. Adverse events may have been under-reported because 
their incidence was based on voluntary reporting. The strength of the study is that BTcP was strictly distinguished from background pain, including its onset, intensity, and duration, as well as the number of BTcP episodes. Adverse reactions were limited and of mild intensity.

\section{Conclusions}

In conclusion, the present study could demonstrate that FBT is an effective and well-tolerated treatment option for BTcP. Still, in Austria, not responding to the first-line treatment with short-acting opioids is mandatory before the cost for rapidonset opioids is covered. Therefore, the evident benefit of FBT has to be transferred into clinical practice, and those formulations should be available without restrictions when indicated. Unsatisfactory management of BTcP that severely affects patients' quality of life should no longer be acceptable.

Acknowledgements Open access funding provided by Medical University of Vienna. We want to thank the patients, investigators, and institutions participating in the current study. We further want to thank Anna Berghoff, MD, PhD for the help in creating the figures.

\section{Compliance with ethical standards}

Conflict of interest TEVA Ratiopharm had no role in the design, conduct, or analysis of the study. Eva Katharina Masel, Robert Landthaler, and Herbert Watzke have received honoraria from Teva Ratiopharm. Margit Gneist is employed by Teva Ratiopharm.

Open Access This article is distributed under the terms of the Creative Commons Attribution-NonCommercial 4.0 International License (http:// creativecommons.org/licenses/by-nc/4.0/), which permits any noncommercial use, distribution, and reproduction in any medium, provided you give appropriate credit to the original author(s) and the source, provide a link to the Creative Commons license, and indicate if changes were made.

\section{References}

1. Anderson R, Saiers JH, Abram S, Schlicht C (2001) Accuracy in equianalgesic dosing. Conversion dilemmas. J Pain Symptom Manag 21:397-406

2. Caraceni A, Portenoy RK (1999) An international survey of cancer pain characteristics and syndromes. IASP Task Force on Cancer Pain International Association for the Study of Pain. Pain 82:263274

3. Chang A, Roeland EJ, Atayee RS, Revta C, Ma JD (2015) Transmucosal immediate-release fentanyl for breakthrough cancer pain: opportunities and challenges for use in palliative care. J Pain Palliat Care Pharmacother 29:247-260

4. Coluzzi PH, Schwartzberg L, Conroy JD, Charapata S, Gay M, Busch MA, Chavez J, Ashley J, Lebo D, McCracken M, Portenoy RK (2001) Breakthrough cancer pain: a randomized trial comparing oral transmucosal fentanyl citrate (OTFC) and morphine sulfate immediate release (MSIR). Pain 91:123-130
5. Davies A, Buchanan A, Zeppetella G, Porta-Sales J, Likar R, Weismayr W, Slama O, Korhonen T, Filbet M, Poulain P, Mystakidou K, Ardavanis A, O'Brien T, Wilkinson P, Caraceni A, Zucco F, Zuurmond W, Andersen S, Damkier A, Vejlgaard T, Nauck F, Radbruch L, Sjolund KF, Stenberg M (2013) Breakthrough cancer pain: an observational study of 1000 European oncology patients. J Pain Symptom Manag 46:619-628

6. Davies AN, Dickman A, Reid C, Stevens AM, Zeppetella G (2009) The management of cancer-related breakthrough pain: recommendations of a task group of the Science Committee of the Association for Palliative Medicine of Great Britain and Ireland. Eur J Pain (London, England) 13:331-338

7. Deandrea S, Montanari M, Moja L, Apolone G (2008) Prevalence of undertreatment in cancer pain. A review of published literature. Ann Oncol 19:1985-1991

8. Farrar JT, Cleary J, Rauck R, Busch M, Nordbrock E (1998) Oral transmucosal fentanyl citrate: randomized, double-blinded, placebo-controlled trial for treatment of breakthrough pain in cancer patients. J Natl Cancer Inst 90:611-616

9. Garnock-Jones KP (2016) Fentanyl buccal soluble film: a review in breakthrough cancer pain. Clin Drug Investig 36:413-419

10. Gomez-Batiste X, Madrid F, Moreno F, Gracia A, Trelis J, Nabal M, Alcalde R, Planas J, Camell H (2002) Breakthrough cancer pain: prevalence and characteristics in patients in Catalonia, Spain. J Pain Symptom Manag 24:45-52

11. Goudas LC, Bloch R, Gialeli-Goudas M, Lau J, Carr DB (2005) The epidemiology of cancer pain. Cancer Investig 23:182-190

12. Kessler J, Bardenheuer HJ (2011) Cancer breakthrough pain. Indications for rapidly effective opioids. Anaesthesist 60:674-682

13. Krakowski I, Theobald S, Balp L, Bonnefoi MP, Chvetzoff G, Collard O, Collin E, Couturier M, Delorme T, Duclos R, Eschalier A, Fergane B, Larue F, Magnet M, Minello C, Navez ML, Richard A, Richard B, Rostaing-Rigattieri S, Rousselot H, Santolaria N, Torloting G, Toussaint S, Vuillemin N, Wagner JP, Fabre N (2003) Summary version of the standards, options and recommendations for the use of analgesia for the treatment of nociceptive pain in adults with cancer (update 2002). Br J Cancer 89(Suppl 1):S67-S72

14. Lennernas B, Frank-Lissbrant I, Lennernas H, Kalkner KM, Derrick R, Howell J (2010) Sublingual administration of fentanyl to cancer patients is an effective treatment for breakthrough pain: results from a randomized phase II study. Palliat Med 24:286-293

15. Lohre ET, Klepstad P, Bennett MI, Brunelli C, Caraceni A, Fainsinger RL, Knudsen AK, Mercadante S, Sjogren P, Kaasa S (2016) From "breakthrough" to "episodic" cancer pain?A European Association for Palliative Care Research Network Expert Delphi Survey Toward a Common Terminology and Classification of Transient Cancer Pain Exacerbations. J Pain Symptom Manage 51:1013-1019

16. Mercadante S, Adile C, Cuomo A, Aielli F, Cortegiani A, Casuccio A, Porzio G (2015) Fentanyl buccal tablet vs. oral morphine in doses proportional to the basal opioid regimen for the management of breakthrough cancer pain: a randomized, crossover, comparison study. J Pain Symptom Manage 50:579-586

17. Mercadante S, Aielli F, Adile C, Costanzi A, Casuccio A (2016) Fentanyl pectin nasal spray versus oral morphine in doses proportional to the basal opioid regimen for the management of breakthrough cancer pain: a comparative study. J Pain Symptom Manage $52: 27-34$

18. Mercadante S, Portenoy RK (2016) Breakthrough cancer pain: twenty-five years of study. Pain 157:2657-2663

19. Oldenmenger WH, Sillevis Smitt PA, van Dooren S, Stoter G, van der Rijt CC (2009) A systematic review on barriers hindering adequate cancer pain management and interventions to reduce them: a critical appraisal. Eur J Cancer (Oxford, England 1990) 45:13701380 
20. Pereira J, Lawlor P, Vigano A, Dorgan M, Bruera E (2001) Equianalgesic dose ratios for opioids. A critical review and proposals for long-term dosing. J Pain Symptom Manag 22:672-687

21. Portenoy RK (2011) Treatment of cancer pain. Lancet 377:22362247

22. Portenoy RK, Payne D, Jacobsen P (1999) Breakthrough pain: characteristics and impact in patients with cancer pain. Pain 81: 129-134

23. Portenoy RK, Taylor D, Messina J, Tremmel L (2006) A randomized, placebo-controlled study of fentanyl buccal tablet for breakthrough pain in opioid-treated patients with cancer. Clin J Pain 22: 805-811

24. Rauck R, North J, Gever LN, Tagarro I, Finn AL (2010) Fentanyl buccal soluble film (FBSF) for breakthrough pain in patients with cancer: a randomized, double-blind, placebo-controlled study. Ann Oncol : Off J Eur Society Med Oncol 21:1308-1314

25. Schug SA, Zech D, Dorr U (1990) Cancer pain management according to WHO analgesic guidelines. J Pain Symptom Manag 5: $27-32$

26. Simon SM, Schwartzberg LS (2014) A review of rapid-onset opioids for breakthrough pain in patients with cancer. J Opioid Manag 10:207-215
27. Smith H (2012) A comprehensive review of rapid-onset opioids for breakthrough pain. CNS drugs 26:509-535

28. Svendsen KB, Andersen S, Arnason S, Arner S, Breivik H, Heiskanen T, Kalso E, Kongsgaard UE, Sjogren P, Strang P, Bach FW, Jensen TS (2005) Breakthrough pain in malignant and nonmalignant diseases: a review of prevalence, characteristics and mechanisms. Eur J Pain(London, England) 9:195-206

29. Vellucci R, Fanelli G, Cortesi PA, Pannuti R, Peruselli C, Romualdi P (2016) Reply-letter to the editor: what to do, and what not to do, when diagnosing and treating breakthrough cancer pain $(\mathrm{BTcP})$ : expert opinion. Drugs 76:1063-1065

30. Ventafridda V, Saita L, Ripamonti C, De Conno F (1985) WHO guidelines for the use of analgesics in cancer pain. Int $\mathrm{J}$ Tissue React 7:93-96

31. Webster LR, Slevin KA, Narayana A, Earl CQ, Yang R (2013) Fentanyl buccal tablet compared with immediate-release oxycodone for the management of breakthrough pain in opioid-tolerant patients with chronic cancer and noncancer pain: a randomized, double-blind, crossover study followed by a 12-week open-label phase to evaluate patient outcomes. Pain Med 14:1332-1345 